\title{
AORTIC VALVE REPLACEMENT WITH THE FREESTYLE STENTLESS BIOPROSTHESIS WITH RESPECT TO SPACIAL ORIENTATION OF PATIENT CORONARY OSTIA
}

\author{
Afksendiyos Kalangos, $\mathrm{MD}, \mathrm{PhD}$, \\ FACA, FETCS \\ Pedro Trigo-Trindade, MD \\ Dominique Vala, MD \\ Aristotelis Panos, MD \\ Bernard Faidutti, MD
}

Objective: This study evaluates our results for safety and efficacy of aortic valve replacement using the Freestyle bioprosthesis (Medtronic, Inc, Minneapolis, Minn) with a new modified subcoronary implantation technique. This technique takes into account the spacial orientation of the stentless bioprosthesis in the aortic root with respect to the patient's coronary ostia rather than the native commissures.

Methods: Fifty-two consecutive patients with predominant aortic valve stenosis underwent aortic valve replacement with a Freestyle bioprosthesis by means of the described modified subcoronary technique over a 15-month period. Fifty of them were followed up by means of echocardiography at discharge, 6 months, and 1 year. There were 19 men and 31 women, with a mean age of $76 \pm 7$ years (range, 58-87 years). Valve size ranged from 21 to $27 \mathrm{~mm}$.

Results: Patients with bicuspid aortic valves had a significantly larger angle between both coronary ostia than patients with tricuspid aortic valves $(P=$ .0001). The peak and mean systolic gradients decreased significantly during the first postoperative year for each valve size $(P \leq .001)$, and the effective valve areas increased significantly during this time interval for each valve size $(P \leq .01)$. Only 13 patients had aortic insufficiency at discharge, which was trivial in 9 and mild in 4 patients. The prevalence of trivial aortic insufficiency decreased during the first postoperative year, and that of mild aortic insufficiency remained unchanged. The sinotubular junction diameter was significantly greater than that of the aortic anulus for each valve size before operation $(P<.001)$. The sinotubular junction diameter decreased significantly after aortic valve replacement and remained unchanged during the first postoperative year for each valve size $(P<.001)$.

Conclusions: Aortic valve replacement with the Freestyle bioprosthesis using the modified subcoronary technique, which takes into account the spacial orientation of the patient's coronary ostia, has hemodynamic results similar to those of other series with different subcoronary implantation techniques. This technique is reproducible, safe at the coronary ostial level, and effective in accommodating variability in angles between human coronary ostia, ranging from $130^{\circ}$ to $170^{\circ}$. Moreover, the great preoperative discrepancies between aortic anulus and sinotubular junction diameters are corrected immediately after operation. (J Thorac Cardiovasc Surg 2000;119:1185-93)
From the Clinic for Cardiovascular Surgery, Division of Cardiology, University Cantonal Hospital of Geneva, Geneva, Switzerland.

Received for publication Aug 12, 1999; revisions requested Oct 7, 1999; revisions received Nov 12, 1999; accepted for publication Jan 10, 2000.

Address for reprints: Afksendiyos Kalangos, MD, PhD, FACA, FETCS, Clinic for Cardiovascular Surgery, University Hospital of Geneva, 24, rue Micheli-du-Crest, 1211 Geneva 14 Switzerland (E-mail: Afksendyios.Kalangos@hcuge.ch).

Copyright () 2000 by The American Association for Thoracic Surgery

0022-5223/2000 $\$ 12.00+0 \quad \mathbf{1 2 / 1 / 1 0 5 6 4 2}$

doi: $10.1067 / \mathrm{mtc} .2000 .105642$ tentless bioprostheses represent a new option in the $\mathcal{S}$ surgical treatment of aortic valvulopathies because of their improved hemodynamic properties. The Freestyle bioprosthesis (Medtronic, Inc, Minneapolis, Minn) is a porcine aortic root that requires implantation techniques similar to those of an aortic homograft, despite different handling characteristics and an inflow covered with Dacron cloth. The subcoronary implantation technique is the most preferred among surgeons, with the most critical issue of this technique being the accommodation of the outflow suture layer with respect to variability in 


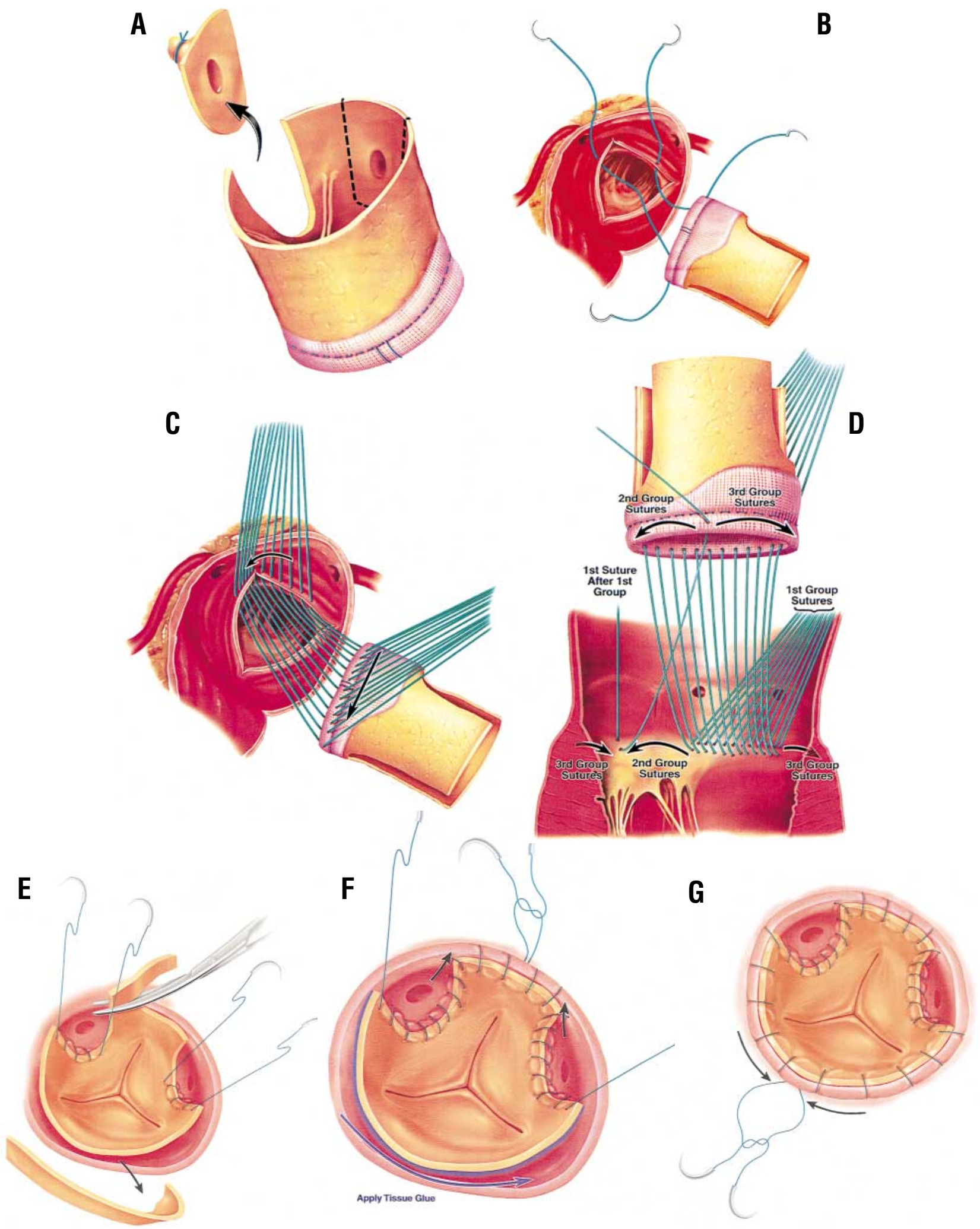

Fig 1. A-G, Surgical technique of subcoronary implantation of a Freestyle bioprosthesis by taking into account the spacial orientation of the patient's coronary ostia. 
position of the human coronary ostia, especially in a dilated aortic root from long-standing aortic stenosis. Although the true prevalence of coronary ostial problems after aortic valve replacement with stentless bioprostheses is unknown, coronary malperfusion could be encountered during or after operation, mainly caused by eventual ligation, trauma, or torsion of the coronary ostia. We describe our experience with a modified subcoronary implantation technique that takes into account the spacial orientation of the patient's coronary ostia rather than that of the commissures. We prospectively evaluated the technique in 50 consecutive patients followed up for a minimum of 1 year.

\section{Patients and methods}

Patient population. Fifty-two consecutive patients underwent elective aortic valve replacement with a Freestyle stentless xenograft for predominantly aortic valve stenosis by means of the technique described hereafter between September 1, 1996, and December 1, 1997. Patients with calcified sinuses of Valsalva, coronary ostia close to the aortic anulus, or a bicuspid aortic valve with opposite coronary ostia were not considered for aortic valve replacement with a stentless bioprosthesis. All patients were operated on by the same surgeon (A.K.). Fifty of them have been prospectively followed up for a minimum of 1 year; one elderly patient died of diffuse bleeding on the third postoperative day, and another died of a ruptured abdominal aortic aneurysm on the fifth postoperative month. There were 19 men and 31 women, whose ages ranged from 58 to 87 years (mean, $76 \pm 7$ years). Eleven patients were assigned to New York Heart Association (NYHA) functional class II, and 39 were assigned to class III. Sixteen patients had pure aortic stenosis, and 34 had additional mild aortic insufficiency. In all patients left ventricular ejection fraction, estimated by echocardiography or contrast ventriculography, was greater than $50 \%$. Nineteen (38\%) patients had concomitant coronary artery disease necessitating simultaneous myocardial revascularization. Two patients underwent concomitant carotid endarterectomy. The sizes of the stentless valves used were $21 \mathrm{~mm}$ in 10 patients, $23 \mathrm{~mm}$ in 12 patients, $25 \mathrm{~mm}$ in 16 patients, and $27 \mathrm{~mm}$ in 12 patients. The mean aortic crossclamp time was $57 \pm 6.5$ minutes for patients with isolated aortic valve replacement and $94.5 \pm 11.3$ minutes for patients with concomitant myocardial revascularization. Nine patients had atrial fibrillation after the operation and were converted to sinus rhythm during the first postoperative month. One patient required a permanent pacemaker for complete heart block before his discharge from the hospital. Forty-nine patients were in sinus rhythm 1 year after the operation. All patients received warfarin sodium during the first 3 postoperative months. In the first postoperative year, 43 patients were assigned to NYHA class I, and 7 were assigned to NYHA class II.

Surgical technique. Cardiopulmonary bypass was established by cannulating the ascending aorta and the right atrium with a 2-stage venous cannula. Antegrade cold blood cardioplegia, topical cooling, and moderate hypothermia to $28^{\circ} \mathrm{C}$ were used for myocardial protection. Cardioplegic solution was delivered either into the aortic root in cases of pure aortic stenosis or selectively into both coronary ostia in the presence of mild aortic insufficiency. A left atrial vent was introduced through the right superior pulmonary vein to maintain a bloodless operative field. The aorta was transversally incised $2 \mathrm{~cm}$ above the right coronary ostium of over two thirds of its circumference to provide good exposure and to maintain symmetry of the aortic root. The native aortic valve was completely excised, and the aortic anulus and anterior leaflet of the mitral valve were extensively debrided of calcium when present. The size of the bioprosthesis was chosen on the basis of the largest sizer that could be smoothly advanced through the anulus. Two marks, corresponding to the left and right coronary ostial positions, were then drawn with a marker on the appropriate valve sizer, and the angle between the two coronary ostia was then measured with a compass. If the measured angle was between $145^{\circ}$ and $155^{\circ}$, the porcine left and right sinuses of Valsalva were trimmed closer to the coronary ostia and down to the cloth skirt, making long, narrower than usual, large scallops because the angle between the two ligated coronary arteries on the Freestyle bioprosthesis corresponds to $150^{\circ} \pm 5^{\circ}$ (Fig 1, A) . If the angle was less than $145^{\circ}$, the scallops were made closer to each other, and if the angle was greater than $155^{\circ}$, they were made farther from each other, according to the extent to which the angle surpassed $150^{\circ} \pm 5^{\circ}$. The noncoronary sinus was left intact. Two stay sutures were placed in the anulus, the first directly below the right coronary ostium and then through the sewing skirt of the Freestyle valve at a point corresponding to the middle of the right scallop, and the second below the left coronary ostium and then through the sewing skirt at a point corresponding to the middle of the left scallop (Fig 1, B). Simple interrupted stitches were then added between these two stay sutures, starting from the stitch below the right coronary ostium, continuing under the right-left coronary commissure, and ending at the stitch below the left coronary ostium (Fig 1, $C$ ). Another stay landmark stitch was placed at the midpoint of the unsutured anulus and then through the midpoint of the unsutured sewing skirt, leaving equal half lengths. Sutures starting from the stitch below the left coronary ostium were added, continuing under the left noncoronary commissure and ending at the landmark point. Another group of sutures was then completed, starting from the stitch below the right coronary ostium, continuing under the right-noncoronary commissure, and ending at the landmark point (Fig 1,D). Interrupted sutures were kept in a horizontal line, except at the level of the membranous septum, where they were passed through the anulus to avoid any injury to the conduction system. The valve was then slid down to the anulus, the knots were tied, and the sutures were cut.

The ouflow suture line was performed by using a doublearmed 5-0 monofilament suture (Premium; Péters Lab, Bobigny, France). Starting below the left coronary ostium, the suture was run up toward the left noncoronary commis- 
Table I. Echocardiographic data

\begin{tabular}{|c|c|c|c|c|}
\hline & \multicolumn{4}{|c|}{ Size of the Freestyle bioprosthesis } \\
\hline & $21 \mathrm{~mm}$ & $23 \mathrm{~mm}$ & $25 \mathrm{~mm}$ & $27 \mathrm{~mm}$ \\
\hline \multicolumn{5}{|c|}{ Aortic valve areas $\left(\mathrm{cm}^{2}\right)$} \\
\hline Discharge & $1.2 \pm 0.1$ & $1.7 \pm 0.1$ & $2.2 \pm 0.2$ & $2.4 \pm 0.1$ \\
\hline $6 \mathrm{mo}$ & $1.2 \pm 0.1$ & $1.7 \pm 0.0$ & $2.2 \pm 0.1$ & $2.4 \pm 0.1$ \\
\hline $1 \mathrm{y}$ & $1.3 \pm 0.1$ & $1.9 \pm 0.1$ & $2.3 \pm 0.1$ & $2.8 \pm 0.2$ \\
\hline$P$ value* & .01 & .0001 & .0001 & .0001 \\
\hline \multicolumn{5}{|c|}{ Mean systolic gradient } \\
\hline Discharge & $13.8 \pm 2.7$ & $10.7 \pm 1.9$ & $8.8 \pm 2.1$ & $10.5 \pm 1.9$ \\
\hline $6 \mathrm{mo}$ & $9.3 \pm 1.8$ & $8.7 \pm 2.7$ & $5.4 \pm 1.4$ & $8.4 \pm 2.9$ \\
\hline $1 \mathrm{y}$ & $8.9 \pm 1.3$ & $7.4 \pm 1.6$ & $4.9 \pm 1.1$ & $4.9 \pm 1.2$ \\
\hline$P$ value* & .001 & .0001 & .0001 & .0001 \\
\hline \multicolumn{5}{|c|}{ Peak systolic gradient } \\
\hline Discharge & $29.4 \pm 5.8$ & $23.6 \pm 2.9$ & $19.8 \pm 3.8$ & $19.6 \pm 2.0$ \\
\hline $6 \mathrm{mo}$ & $18.4 \pm 4.1$ & $18.9 \pm 2.0$ & $11.9 \pm 2.6$ & $14.3 \pm 2.0$ \\
\hline $1 \mathrm{y}$ & $13.3 \pm 2.0$ & $18.9 \pm 2.4$ & $11.4 \pm 2.3$ & $11.4 \pm 2.0$ \\
\hline$P$ value* & .0001 & .0004 & .0001 & .0001 \\
\hline
\end{tabular}

*Values at discharge compared with values at 1 year.

sure, and the second needle was then taken and run up toward the left-right coronary commissure. A second stitch was placed under the right coronary ostium in a similar fashion, and the suture was run up toward the left-right coronary commissure, after which the two sutures were tied outside the aorta. The second needle was taken, and the suture was run up toward the right noncoronary commissure. If necessary, the outflow edge of the prosthesis was trimmed to correspond to the height of the aortotomy (Fig 1,E). The space behind the noncoronary sinus was filled with tissue glue (Fig 1,F), and the suture was then run from each side to the center of the noncoronary sinus and tied outside the aortic wall (Fig 1, $G$ ).

Echocardiographic measurements. Transthoracic echocardiography was performed before the operation and at 6 and 12 months after the operation. Examination included 2dimensional, 2-dimensional-derived M-mode, continuous wave, and pulsed Doppler and color Doppler analyses by using a Hewlett-Packard Sonos 2500E echocardiographic machine (Hewlett-Packard Inc, Palo Alto, Calif) with a 2.5$\mathrm{MHz}$ transducer. Left parasternal, apical, right parasternal, subcostal, and suprasternal standard views were obtained in a step-by-step successive pattern of investigation. All measurements were taken by the same examiner with the patient in a hemodynamically stable condition, and variables were calculated with the use of previously reported formulas. ${ }^{1}$ All variables were averaged from 5 cardiac cycles in sinus rhythm and from 10 cardiac cycles if atrial fibrillation was present. The parasternal long-axis view was used to obtain sizing of the aortic root at the level of the aortic anulus and the sinotubular junction and was averaged from 5 measurements at end diastole.

Statistical analysis. Variables were expressed as means \pm SD. Statistics were calculated by using the SAS statistical package (SAS Institute, Inc, Cary, NC). A paired $t$ test was used to determine whether there were significant changes in mean gradient, peak gradient, and aortic valve area from dis- charge to the 1-year postoperative evaluation. The Spearman rank correlation coefficient was calculated to determine the correlation between the aortic anulus diameter measured by use of 2-dimensional echocardiography and the actual valve size. A paired $t$ test was also used to compare preoperative values of the aortic anulus and the sinotubular junction diameters for each valve size. Both tests were adjusted for multiple comparisons. Repeated-measures analysis of variance was used to determine whether there was a significant change in sinotubular junction diameter across time. A $t$ test was used to compare the angle between the two coronary ostia between the bicuspid and tricuspid aortic valves.

\section{Results}

Angles between both patient coronary ostia and location of coronary ostia with respect to commissures. In 16 patients the angle between the two coronary ostia was between $145^{\circ}$ and $155^{\circ}$, in 20 patients it was inferior to $145^{\circ}$, and in 14 patients it was superior to $155^{\circ}$. In all of the 11 patients with a bicuspid aortic valve, the mean angle between the two coronary ostia was $162^{\circ} \pm 9^{\circ}$, and in all of the 39 patients with a tricuspid aortic valve, it was $143^{\circ} \pm 10^{\circ}$. Patients with bicuspid aortic valves had significantly larger angles between the coronary ostia than patients with tricuspid aortic valves $(P=.0001)$. In 8 patients with tricupid aortic valves, the right coronary ostium was closer to the right noncoronary commissure, and the left coronary ostium was in the middle of the left sinus of Valsalva. In 2 patients the right coronary ostium was closer to the right noncoronary commissure, and the left coronary ostium was closer the right-left coronary commissure. In 3 patients the left coronary ostium was 


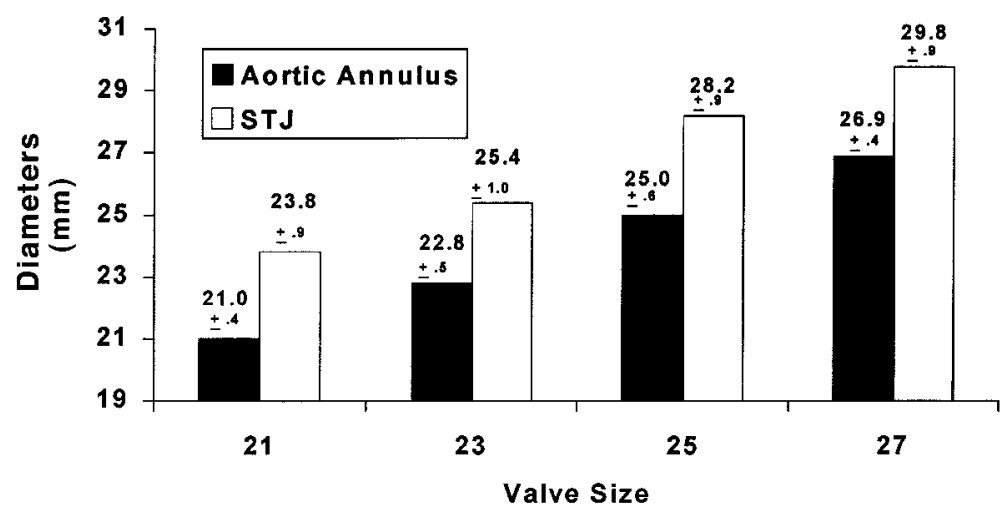

Fig 2. Aortic anulus and sinotubular junction (STJ) diameters obtained by means of 2-dimensional transthoracic echocardiography before the operation for each valve size.

closer to the right noncoronary commissure, and the right coronary ostium was in the middle of the right sinus of Valsalva. In 1 patient the left coronary ostium was closer to the left noncoronary commissure, and the right coronary ostium was in the middle of the right coronary sinus. In patients with bicuspid aortic valves, the right coronary ostium was closer to the anterior commissure.

Effective valve area and peak and mean pressure gradients. Table I shows the effective valve areas and the mean and peak systolic gradients, respectively, obtained by means of Doppler echocardiography for each valve size at discharge and at 6 months and 1 year postoperatively. The effective orifice areas increased significantly during the first 12 months after implantation for each valve size $(P=.01$ for valve size of 21 $\mathrm{mm} ; P=.0001$ for valve sizes of 23,25 , and $27 \mathrm{~mm}$ ). The mean and peak gradients across the stentless valve decreased significantly during the first postoperative year for each valve size $(P \leq .001)$.

Aortic anulus and sinotubular junction diameters. Fig 2 shows the aortic anulus and sinotubular junction diameters obtained by using transthoracic echocardiography 1 month before the operation for each valve size. The correlation between the aortic anulus diameters measured by 2-dimensional echocardiography and the actual valve sizes implanted at the operation was excellent and statistically significant $(P=.0001)$, with a correlation coefficient of $96.6 \%$ (Fig 3 ). Table II provides the distribution of aortic root measurements for each valve size. The sinotubular junction diameter was significantly greater than that of the aortic anulus before the operation for each valve size $(P=.0001)$. It exceeded that of the aortic anulus by $1 \mathrm{~mm}$ in 2 patients, $2 \mathrm{~mm}$ in 23 patients, $3 \mathrm{~mm}$ in 17 patients, and $4 \mathrm{~mm}$ in 8 patients. The sinotubular junction diameter decreased significantly after the operation and remained unchanged during the first postoperative year for each valve size $(P=.0001$, Fig 4$)$.

Presence of aortic insufficiency. Aortic insufficiency was graded trivial in 9 and mild in 4 patients at discharge, trivial in 8 and mild in 4 patients at 6 months, and trivial in 4 and mild in 4 patients at 1 year. The prevalence of trivial aortic insufficiency decreased, and that of mild aortic insufficiency did not change with time (Fig 5). The fact that only a few patients had trivial or mild aortic insufficiency prevented us from accurately performing a $\chi^{2}$ test.

\section{Discussion}

Several recent studies regarding the use of new stentless bioprostheses as aortic valve substitutes have demonstrated improved hemodynamics as a result of higher effective orifice areas resulting in lower residual pressure gradients and rapid resolution of left ventricular hypertrophy. ${ }^{2-6}$ Their more physiologic flow profiles promise improved durability with mitigation from early calcification through new antimineralization and fixation treatments. ${ }^{7}$ Among the multiple current stentless bioprostheses, the Freestyle bioprosthesis is presented as an intact porcine aortic root with ligated coronary arteries. Its methods of implantation, known as the full root, root inclusion, and subcoronary techniques, are not dissimilar to those of a homograft. The subcoronary technique in which 2 or 3 sinuses of the bioprosthesis are completely excised is the most popular among surgeons, although the full root and root-inclusion techniques seem to be hemodynamically superior. ${ }^{8,9}$ 


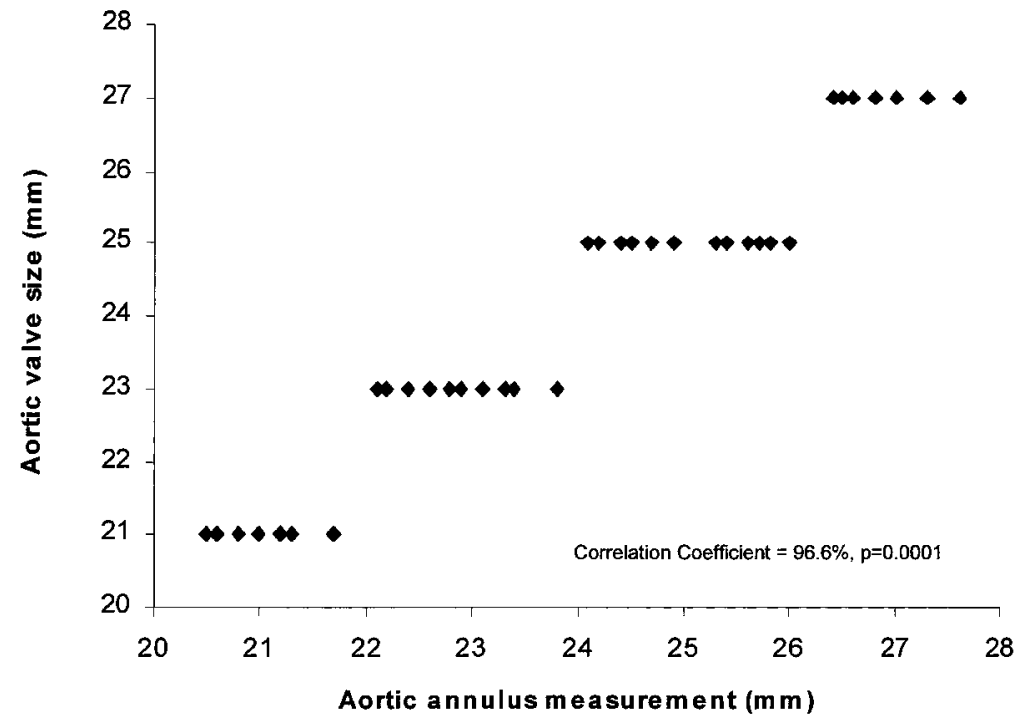

Fig 3. Correlation between aortic anulus measurements obtained by means of 2-dimensional echocardiography and implanted aortic valve size.

Table II. Aortic anulus measurements obtained by means of echocardiography compared with aortic valve size

\begin{tabular}{lccccc}
\hline & \multicolumn{4}{c}{ Aortic anulus measurements } \\
\cline { 2 - 5 } Valve size & $n$ & Mean & SD & Minimum & Maximum \\
\hline $21 \mathrm{~mm}$ & 10 & 21.0 & 0.4 & 20.5 & 21.7 \\
$23 \mathrm{~mm}$ & 12 & 22.8 & 0.5 & 22.1 & 23.8 \\
$25 \mathrm{~mm}$ & 16 & 25.0 & 0.6 & 2.1 & 26.0 \\
$27 \mathrm{~mm}$ & 12 & 26.9 & 0.4 & 26.4 & 27.6 \\
\hline
\end{tabular}

From a technical point of view, the subcoronary technique, as with the two other techniques, is focused on the spacial orientation of the porcine commissures by aligning them with the patient's aortic commissures at the inflow insertion level and by facing openings created on the porcine aortic sinuses to native coronary ostia at the outflow insertion level. This necessary 2-layer suture at the inflow and outflow levels for insertion of a stentless bioprosthesis renders its implantation technically more demanding than the attachment of the sewing ring of a stented bioprosthesis to the patient's valvular anulus by a single suture layer.

Suturing is considered particularly dangerous at the outflow level in the depth of the left and right sinuses of Valsalva, especially for surgeons who have limited experience in this field. Moreover, the wide variation in the angle $\left(90^{\circ}-180^{\circ}\right)$ between human coronary ostia and in the location of the ostia with respect to the commissures and the aortic anulus may increase the difficulty of orienting the Freestyle bioprosthesis into the aortic root in relation to the coronary ostia, especially in patients with long-standing severe aortic stenosis in whom aortic root geometry becomes highly variable. Efforts usually concentrated on adapting variable angles between human coronary ostia and their location with respect to commissures to the largely scalloped porcine left and right coronary sinuses of Valsalva only at the outflow insertion level ${ }^{10-12}$ become more difficult, especially in cases of native coronary ostia situated closer to the commissures than to the centers of the sinuses of Valsalva. Implantation techniques must take into account the alignment of native and porcine commissures at the inflow level. Coronary ostia were located closer to the commissures in one third of our patients, hence impeding the appropriate implantation of a stentless bioprosthesis without creating distortion of the valve tissue at the outflow insertion level, even in cases of extensively trimmed porcine sinuses of Valsalva. This fact was the main reason that we developed and applied the above-described implan- 


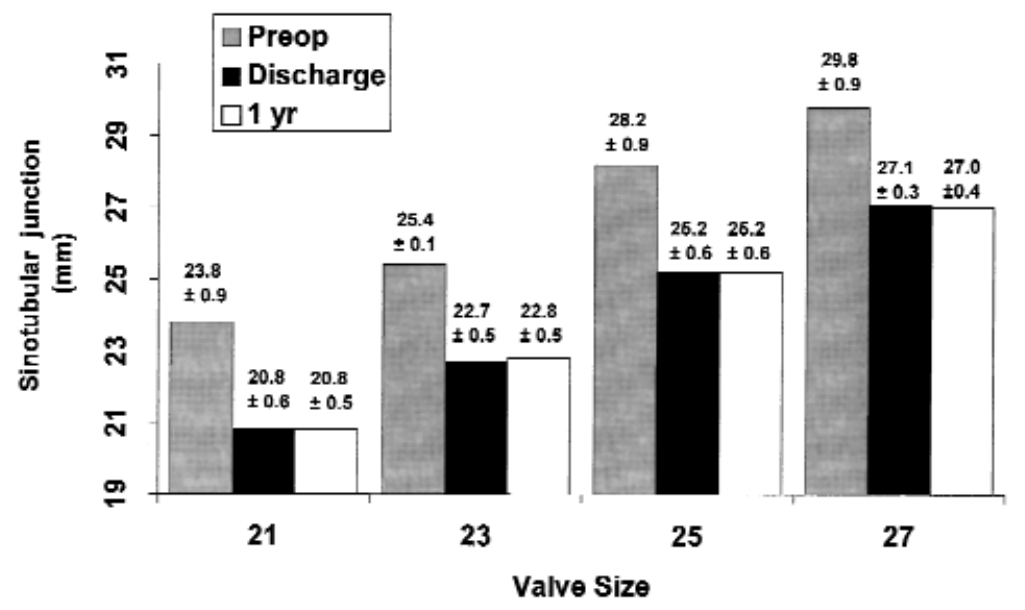

Fig 4. Evolution of the sinotubular junction diameter during the first postoperative year for each valve size. Echocardiographic controls show that the sinotubular junction diameter decreased significantly for each valve size at discharge $(P<.001$ vs before the operation) and remained unchanged when compared with values at 1 year postoperatively.

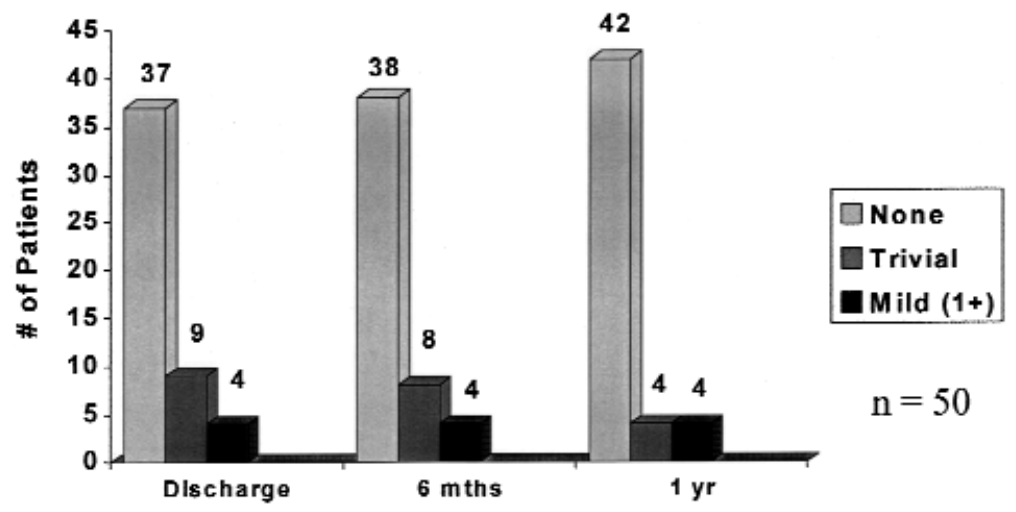

Fig 5. Evolution of residual aortic insufficiency during the first postoperative year.

tation technique by taking into account the spacial orientation of the patient's coronary ostia rather than that of his or her commissures at the inflow insertion level. We believe this technique avoids not only eventual problems at the coronary ostial level by ensuring perfect alignment between the economically excised Ushaped portions of the porcine aortic sinuses and human coronary ostia but also the distortion of valve tissue at the inflow insertion level, which can lead to early valve failure. ${ }^{13}$ In addition, we believe that this implantation technique is more secure for bicuspid aortic valves in which the spacial configuration of commissures is usually abnormal and angles between coronary ostia are larger than those of tricuspid aortic valves, as demonstrated in our series. This situation can sometimes perturb surgeons, who lack key implanta- tion points. Trimming the porcine sinuses of Valsalva closer to or farther from each other according to values smaller or greater than the angle of porcine coronary ostia on the Freestyle bioprosthesis $\left(150^{\circ} \pm 5^{\circ}\right)$ allows more secure implantation by avoiding the crimping of the anulus in attempts to accommodate smaller or greater human coronary ostial angles to the porcine coronary ostia. However, we consider angles smaller than $130^{\circ}$ and greater than $170^{\circ}$ to constitute a major drawback for our implantation technique. They induce crimping of the sewing skirt at its closest perimeter between the two aligned coronary ostia and that of the remaining part of the aortic anulus when the angle between the patient's coronary ostia is smaller than $130^{\circ}$. They also result in crimping of the aortic anulus at its closest perimeter between the aligned coronary 
ostia and that of the remaining part of the sewing skirt when the angle between the patient's coronary ostia is greater than $170^{\circ}$. This condition can create stenosis at the inflow level, and it is for this reason that we believe the implantation of any kind of stentless bioprosthesis becomes questionable for angles smaller than $130^{\circ}$ and greater than $170^{\circ}$, even with larger scalloped porcine sinuses of Valsalva.

Our study shows that the implantation of a Freestyle stentless bioprosthesis according to this modified subcoronary implantation technique gives results concordant with echocardiographic variables of previous studies on the basis of different subcoronary techniques. ${ }^{10,11,14}$ The very low prevalence of residual aortic regurgitation and postoperative effective orifice areas similar to those of other series in which different subcoronary implantation techniques were used demonstrates the safety and effectiveness of our implantation technique. The implantation method we describe here is reproducible, safe at the coronary ostial level, and effective in accommodating variability in angles between human coronary ostia ranging from $130^{\circ}$ to $170^{\circ}$. Moreover, the act of excising a longitudinal segment from the porcine aortic sinuses, narrower than that required by other subcoronary implantation techniques, renders our technique comparable with that of a root inclusion technique, despite the eventual risk of coronary ostial stenosis by intimal hyperplasia. Correction of great discrepancies between the aortic anulus and sinotubular junction diameters immediately after the operation and its maintenance over the first postoperative year in our series proves that the patient's aortic root is completely adapted to the physiologic geometry of the porcine aortic root, which retains its shape as in a root inclusion technique. This fact demonstrates that there is no need for reducing the sinotubular junction as formerly proposed when using 3 scalloped stentless bioprostheses, in which the correction of a larger sinotubular junction diameter-present in almost all cases of long-standing aortic stenosis-is mandatory for preventing recurrence of aortic regurgitation caused by poor coaptation and increased stress on the leaflets. ${ }^{1}$

We thank Mrs Katherine Johnson for her statistical support and Mrs Ann Prosser and Mrs Huguette Sollero for their help in preparing this manuscript.

\section{REFERENCES}

1. David TE, Feindel CM, Bos J, Sun Z, Scully HE, Rakowski H. Aortic valve replacement with a stentless porcine aortic valve: a six-year experience. J Thorac Cardiovasc Surg 1994;108:1030-6.

2. Sintek CF, Fletcher AD, Khonsari S. Stentless porcine aortic root:
Valve of choice for the elderly patient with a small aortic root? J Thorac Cardiovasc Surg 1995;109:871-6.

3. Christakis GT, Joyner CD, Morgan CD, Fremes SE, Buth KJ, Sever JY, et al. Left ventricular mass regression early after aortic valve replacement. Ann Thorac Surg 1996;62:1084-9.

4. Del Rizzo DF, Goldman BS, Christakis GT, David TE. Hemodynamic benefits of the Toronto stentless valve. J Thorac Cardiovasc Surg 1996;112:1431-46.

5. Jin XY, Zhang ZM, Gibson DG, Yacoub MH, Pepper JR. Effects of valve substitute on changes in left ventricular function and hypertrophy after aortic valve replacement. Ann Thorac Surg 1996;62:683-90.

6. Westaby S, Amarasena N, Long V, Prothero A, Amarasena GA, Banning AP, et al. Time-related hemodynamic changes after aortic replacement with the Freestyle stentless xenograft. Ann Thorac Surg 1995;60:1633-9.

7. Walther T, Falk V, Autschbach R, Diegeler A, Rauch T, Weigl $\mathrm{C}$, et al. Comparison of different anticalcification treatments for stentless bioprostheses. Ann Thorac Surg 1998;66(Suppl 6):429-54.

8. Yoganathan AP, Eberhardt CE, Walker PG. Hydrodynamic performance of the Medtronic Freestyle aortic root bioprosthesis. J Heart Valve Dis 1994;3:571-80.

9. Kon ND, Westaby S, Amarasena N, Pillai R, Cordell AR. Comparison of implantation techniques using Freestyle stentless porcine aortic valve. Ann Thorac Surg 1995;59:857-62.

10. Westaby S, Amarasena N, Ormerod O, Amarasena GA, Pillai R. Aortic valve replacement with the Freestyle stentless xenograft. Ann Thorac Surg 1995;60:S422-7.

11. Doty JR, Flores JH, Millar RC, Doty DB. Aortic valve replacement with Medtronic Freestyle bioprosthesis: operative technique and results. J Cardiac Surg 1998;13:208-17.

12. Krause AH Jr. Technique for complete subcoronary implantation of the Medtronic Freestyle porcine bioprosthesis. Ann Thorac Surg 1997;64:1495-8.

13. Vesely I, Boughner D, Song T. Tissue buckling as a mechanism of bioprosthetic valve failure. Ann Thorac Surg 1988;46:302-8.

14. Dumesnil JG, LeBlanc MH, Cartier PC, Métras J, Desaulniers D, Doyle DP, et al. Hemodynamic features of the Freestyle aortic bioprosthesis compared with stented bioprosthesis. Ann Thorac Surg 1998;66:S130-3.

\section{Commentary}

This article describes a modification of the subcoronary implant technique for the Freestyle stentless xenograft, which the authors suggest may reduce the risk of native coronary ostial occlusion. The method involves measurement of the angle between the native coronary ostia to prejudge the extent of scalloping the porcine coronary sinuses. The authors state that patients with adverse root anatomy, including low takeoff of the native coronary arteries, root calcification, or substantial discrepancy between the anulus and sinotubular junction diameters, were not considered for implantation of the Freestyle valve.

There are a number of criticisms of the article as presented. First, the authors suggest that the original sub- 